\title{
Single-Molecule Kinetics of Nanoparticle Catalysis
}

\author{
Weilin $\mathrm{Xu}^{\dagger}$, Hao Shen, Guokun Liu, and Peng Chen $(\varangle)$ \\ Department of Chemistry and Chemical Biology, Cornell University, Ithaca, NY 14853, USA \\ ${ }^{\dagger}$ Present address: Department of Chemical Engineering, University of California, Berkeley, CA 94720, USA \\ Received: 5 September 2009 / Revised: 28 October 2009 / Accepted: 29 October 2009 \\ CTsinghua University Press and Springer-Verlag 2009. This article is published with open access at Springerlink.com
}

\begin{abstract}
Owing to their structural dispersion, the catalytic properties of nanoparticles are challenging to characterize in ensemble-averaged measurements. The single-molecule approach enables studying the catalysis of nanoparticles at the single-particle level with real-time single-turnover resolution. This article reviews our single-molecule fluorescence studies of single Au-nanoparticle catalysis, focusing on the theoretical formulations for extracting quantitative reaction kinetics from the single-turnover resolution catalysis trajectories. We discuss the singlemolecule kinetic formulism of the Langmuir-Hinshelwood mechanism for heterogeneous catalysis, as well as of the two-pathway model for product dissociation reactions. This formulism enables the quantitative evaluation of the heterogeneous reactivity and the differential selectivity of individual nanoparticles that are usually hidden in ensemble measurements. Extension of this formulism to single-molecule catalytic kinetics of oligomeric enzymes is also discussed.
\end{abstract}

\section{KEYWORDS}

Single-nanoparticle catalysis, single-molecule fluorescence detection, Langmuir-Hinshelwood mechanism, reactivity heterogeneity, parallel reaction pathways, differential selectivity

\section{Introduction}

With the ever-increasing demands for energy and declining reserves of fossil fuels, efficient use of fossil fuels and energy extraction from alternative feedstocks are critical for mankind's sustainable future. Catalysis is one of the key technologies capable of helping to meet this energy challenge. Nanoparticle catalysts are an integral part of catalysis technology, and they can catalyze many energy conversion reactions, often more efficiently than their bulk counterparts [1-4]. With the rapid advances in nanoscience, new nanoparticle catalysts and novel catalytic properties continue to emerge [3, 5-7].

A tremendous amount of work has been done in characterizing the catalytic properties of nanoparticles at the ensemble level, where a collection of nanoparticles are studied simultaneously; significant insights have been obtained into the structureactivity correlations of nanoparticle catalysts. These ensemble-averaged characterizations are inadequate, however, as nanoparticle catalysts - except those molecular metal clusters that have well-defined chemical stoichiometry - have structural dispersions, which inevitably lead to different properties for individual particles. But how different are they? Are

Address correspondence to pc252@cornell.edu 
the differences significant?

To address these questions, one needs to study nanoparticle catalysis at the single-particle level. Several research groups have carried out electrochemical measurements on a single nanoelectrode or nanoparticle by detecting electrical current [8-13]. Using surface plasmon spectroscopy, Novo et al. observed redox catalysis by single Aunanocrystals [14].

Our group has developed a single-molecule fluorescence approach to study nanoparticle catalysis at the single-particle level, using Aunanoparticles as exemplary catalysts [15-18]. Rapid technological advances have made it possible to detect the fluorescence of a single molecule readily under ambient conditions. By detecting a fluorescent product of a catalytic reaction, we can monitor the catalysis of individual colloidal Au-nanoparticles in real time at single-turnover resolution under ambient solution conditions. In this way, we were able to gain quantitative insight into the heterogeneous reactivity, differential selectivity between parallel reaction pathways, surface-restructuring-coupled catalytic dynamics, and reactant-concentration-dependent surface switching behaviors of nanoparticle catalysts. This single-molecule fluorescence approach builds on the pioneering work in single-enzyme studies [19-22], and was recently also employed in studying microand nano-scale solid catalysts [23-25]. In this article, we briefly review our experiments and focus our discussion on the theoretical analysis of the singlemolecule data for extracting the kinetics of singlenanoparticle catalysis. An earlier review focused on the experimental examinations of heterogeneous and dynamic behaviors of single nanoparticles [18].

\section{Single-molecule detection of single- nanoparticle catalysis}

Our approach is based on single-molecule microscopy of fluorogenic reactions. Figure 1(a) depicts our experimental design using total internal reflection fluorescence microscopy [15, 17]. We immobilize individual Au-nanoparticles on a quartz slide at low density inside a flow cell, so that individual particles are well separated by many micrometers, a scale much larger than the diffractionlimited spatial resolution ( half a micron) in optical microscopy. We design a reaction, catalyzed by the Au-nanoparticles, which converts a non-fluorescent substrate molecule to a highly fluorescent product. With the substrate solution flowing above the quartz surface, catalysis occurs on the Au-nanoparticle surfaces. Every reaction catalyzed by a single Aunanoparticle generates a fluorescent product. Under constant laser illumination, every product molecule gives out intense fluorescence signals. By detecting the fluorescence of the product one molecule at a time, we can monitor the catalytic reactions by a single Au-nanoparticle in real time at single-reaction (i.e., single-turnover) resolution. The total internal reflection geometry of laser excitation confines the excitation within 100-200 $\mathrm{nm}$ above the quartz surface, reducing the background and facilitating the single-molecule fluorescence detection. Our specific reaction is the Au-nanoparticle catalyzed reduction of resazurin to resorufin by $\mathrm{NH}_{2} \mathrm{OH}$ in aqueous

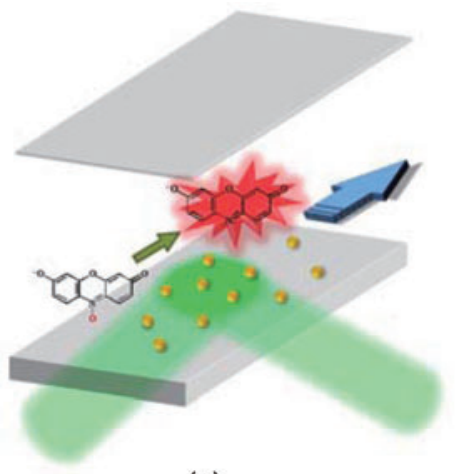

(a)

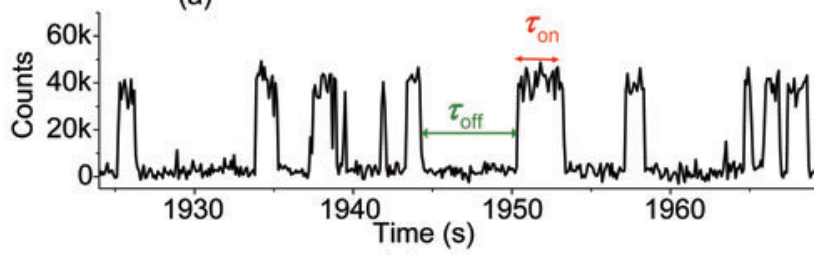

(c)

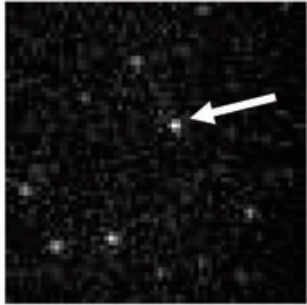

(b)
Figure 1 (a) Experimental scheme for the use of total internal reflection fluorescence microscopy and a flow cell to image catalytic turnovers of individual Au-nanoparticles. Au-nanoparticles (golden balls) are immobilized on the quartz slide. The reactant solution is flowed on top. The fluorescence of the catalytic product is excited by a 532-nm laser in total internal reflection geometry. (b) Typical image $(\sim 18 \mu \mathrm{m} \times 18 \mu \mathrm{m})$ of fluorescent products during catalysis by $6-\mathrm{nm}$ Au-nanoparticles. (c) Segment of the fluorescence time trajectory from the fluorescence spot marked by the arrow in (b). Figures adapted from $\mathrm{Xu}$ et al. $[15,17]$ 
solutions. The highly fluorescent product resorufin is the target of single-molecule detection.

Figure 1 (b) shows a typical fluorescence image from a real-time movie recorded by an electron multiplying charge-coupled device (EMCCD) camera at the $100-\mathrm{ms}$ frame rate. The image shows localized bright spots, which are fluorescence signals of the product molecules adsorbed on individual Aunanoparticles. A typical fluorescence time trajectory from one such bright spot, i.e., one Au-nanoparticle, contains stochastic off-on fluorescence bursts (Fig. $1(\mathrm{c})$ ). The digital nature of the trajectory and the consistent height of its on-level are characteristic of single-molecule fluorescence detection. Each sudden intensity increase in the trajectory marks a product formation event on a nanoparticle. The product molecule stays on the nanoparticle for a while due to its finite affinity for the nanoparticle surface, before it dissociates; the dissociation is marked by a sudden intensity decrease in the trajectory. Every off-on cycle in the trajectory corresponds to a single turnover of a catalytic formation of a product and its subsequent dissociation on one nanoparticle. The actual chemical transformations in the catalytic cycle occur at subpicosecond timescales and are irresolvable in these single-molecule fluorescence trajectories. The fluorescence blinking of the product molecule has no significant contribution here, as it happens on much slower timescales [15]. Once the product molecule leaves the nanoparticle surface, it becomes undetectable due to its fast diffusion and is carried away by the solution flow. Occasionally, multiple on-levels are observed in the trajectory, indicating a new product molecule is formed on the nanoparticle before an earlier one dissociates away; these multilevel events are rare because the on-times are much shorter on average than the off-times [15].

In a single-particle fluorescence turnover trajectory, the waiting times, $\tau_{\text {off }}$ and $\tau_{\text {on }}$, are the two most important observables (Fig. 1(c)). Their clean resolution separates the catalysis into two parts temporally: $\tau_{\text {off }}$ is the waiting time before each product formation, and $\tau_{\text {on }}$ is the waiting time for product dissociation after its formation. The individual values of $\tau_{\text {off }}$ and $\tau_{\text {on }}$ are stochastic, but their statistical properties, such as their distributions and averages, are defined by the underlying reaction kinetics [16]. By analyzing how the statistical properties of $\tau_{\text {off }}$ and $\tau_{\text {on }}$ depend on the resazurin concentration [S], we have formulated a LangmuirHinshelwood mechanism for the catalytic conversion reaction and a two-pathway mechanism for the product dissociation reaction [15]. In the following, we discuss the kinetic mechanism for the Aunanoparticle catalysis and the single-molecule kinetic formulism that connects the mechanism to the statistical properties of $\tau_{\text {off }}$ and $\tau_{\text {on }}$. We use the results of 6-nm pseudo-spherical Au-nanoparticles as illustrations.

\section{Single-molecule kinetic theory of nanoparticle catalysis}

Figure 2 shows the kinetic mechanism for Aunanoparticle catalyzed reduction of resazurin to resorufin [15]. The catalytic product formation reaction follows a Langmuir-Hinshelwood mechanism, in which the nanoparticle catalyzes the substrate conversion to product while maintaining a fast substrate adsorption equilibrium (reaction (i) in Fig. 2). After being generated, the product can dissociate via two parallel pathways: one a substrate-assisted pathway, in which the nanoparticle binds a substrate first before the product leaves the nanoparticle surface (reactions (ii) and (iii)), the other a direct dissociation pathway (reaction (iv)). The fluorescence state (off or on) of the nanoparticle is indicated at each reaction stage. The contribution of $\mathrm{NH}_{2} \mathrm{OH}$ is omitted in the mechanism as a simplification, as it is kept at a saturating concentration in our experiments and is thus not a rate-limiting reagent $[15,17]$. In the following, we discuss how to derive the single-molecule kinetic equations that connect this mechanism to the statistical properties of $\tau_{\text {off }}$ and $\tau_{\text {on }}$ in the singleparticle turnover trajectories.

\section{1 $\tau_{\text {off }}$ reaction: Langmuir-Hinshelwood mechanism for catalytic product formation}

$\tau_{\text {off }}$ is the waiting time before each product formation. At the onset of $\tau_{\text {off }}$ no product has yet formed on the nanoparticle surface; once a product is formed, 


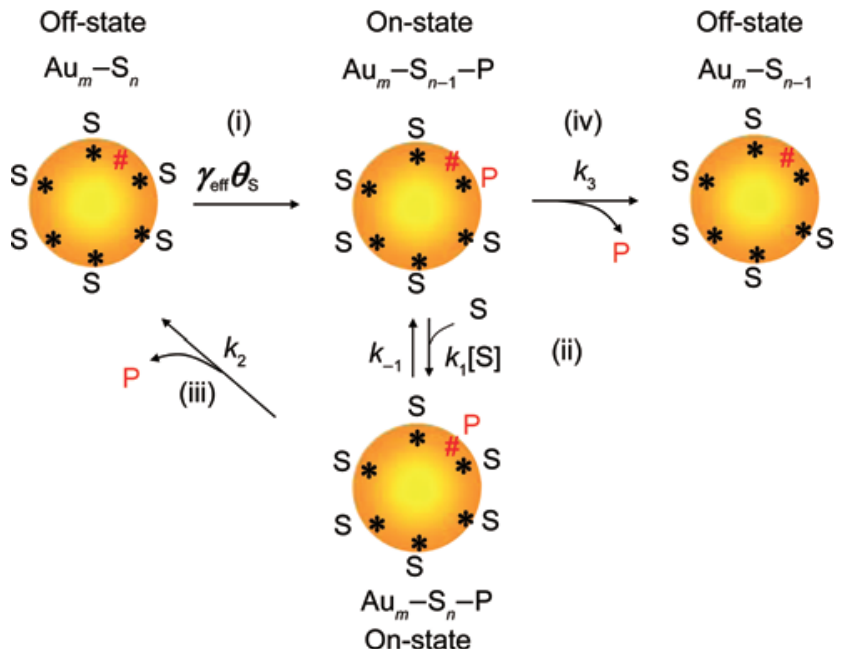

Figure 2 Kinetic mechanism of Au-nanoparticle catalysis. $\mathrm{Au}_{m}$ : $\mathrm{Au}-$ nanoparticle; $S$ : the substrate resazurin; $P$ : the product resorufin; [S]: substrate concentration. $\mathrm{Au}_{m} \mathrm{~S}_{n}$ represents an Au-nanoparticle having $n$ adsorbed substrate molecules. The fluorescence state (on or off) of the nanoparticle is indicated at each reaction stage. $\gamma_{\text {eff }}$ $=k n_{\mathrm{T}}$ and represents the combined reactivity of all surface catalytic sites of a nanoparticle. $k$ is a rate constant representing the reactivity per catalytic site for the catalytic conversion. $n_{\mathrm{T}}$ is the total number of surface catalytic sites on one Au-nanoparticle. $\theta_{s}$ is the fraction of catalytic sites that are occupied by substrates and equals $K_{1}[S] /$ $\left(1+K_{1}[S]\right)$, where $K_{1}$ is the substrate adsorption equilibrium constant. This kinetic mechanism is formulated at saturating concentrations of the co-substrate $\mathrm{NH}_{2} \mathrm{OH}$, whose contribution is not included explicitly as an approximation. Figure taken from $\mathrm{Xu}$ et al. [15]

$\tau_{\text {off }}$ ends and $\tau_{\text {on }}$ starts. Based on the LangmuirHinshelwood mechanism in Fig. 2, the process taking place during $\tau_{\text {off }}$ is reaction (i) reproduced as Eq. (1) below:

$$
\begin{aligned}
& \mathrm{Au}_{m} \mathrm{~S}_{n} \stackrel{\gamma_{\text {app }}}{\longrightarrow} \mathrm{Au}_{m} \mathrm{~S}_{n-1}-\mathrm{P} \\
& \text { Off-state } \quad \text { On-state }
\end{aligned}
$$

where $n$ is the number of substrate resazurin molecules adsorbed on the nanoparticle surface, $\mathrm{Au}_{m}$ stands for the Au-nanoparticle, $\mathrm{S}$ for the substrate resazurin, $\mathrm{P}$ for the product resorufin, and $\gamma_{\text {app }}$ is the apparent rate constant for forming one product on the surface of one nanoparticle. Here the formation of one $\mathrm{P}$ molecule and thus of the state $\mathrm{Au}_{m} \mathrm{~S}_{n-1}-\mathrm{P}$ marks the completion of a $\tau_{\text {off }}$. From LangmuirHinshelwood kinetics for heterogeneous catalysis [26], $\gamma_{\text {app }}$ takes the form:

$$
\gamma_{\mathrm{app}}=k n
$$

where $k$ is the rate constant representing the intrinsic reactivity per catalytic site for the catalytic conversion reaction. From the Langmuir adsorption isotherm [26],

$$
n=n_{\mathrm{T}} \theta_{\mathrm{s}}=n_{\mathrm{T}} \frac{K_{1}[\mathrm{~S}]}{1+K_{1}[\mathrm{~S}]}
$$

where $n_{\mathrm{T}}$ is the total number of surface catalytic sites on one nanoparticle, $\theta_{\mathrm{S}}$ is the fraction of catalytic sites occupied by the substrate, and $K_{1}$ is the substrate adsorption equilibrium constant. Then we have

$$
\gamma_{\text {app }}=k n_{\mathrm{T}} \theta_{\mathrm{s}}=\frac{\gamma_{\text {eff }} K_{1}[\mathrm{~S}]}{1+K_{1}[\mathrm{~S}]}
$$

where $\gamma_{\text {eff }}=k n_{\mathrm{T}}$ and represents the combined reactivity of all surface catalytic sites on one $\mathrm{Au}$ nanoparticle.

In conventional ensemble measurements where reactions of a large number of colloidal Au-nanoparticles are measured in solution simultaneously, the kinetic rate equation for the reaction in Eq. (1) is

$$
\frac{\mathrm{d}\left[\mathrm{Au}_{m} \mathrm{~S}_{n-1}-\mathrm{P}\right]}{\mathrm{d} t}=-\frac{\mathrm{d}\left[\mathrm{Au}_{m} \mathrm{~S}_{n}\right]}{\mathrm{d} t}=\gamma_{\mathrm{app}}\left[\mathrm{Au}_{m} \mathrm{~S}_{n}\right]
$$

where $\left[\mathrm{Au}_{m} \mathrm{~S}_{n}\right]$ is the concentration of Aunanoparticles that do not carry any product, and $\left[\mathrm{Au}_{m} \mathrm{~S}_{n-1}-\mathrm{P}\right]$ is the concentration of Au-nanoparticles on which one product molecule is generated. Here the approximation of $\gamma_{\text {app }}$ as a pseudo-first-order rate constant is valid, provided [S] is time-independent (see below).

In single-nanoparticle measurements, although the concentration of the substrate, [S], is still a valid description, the concentration of one nanoparticle is meaningless and each nanoparticle has a certain probability of being in either the $A u_{m} S_{n}$ or the $A u_{m} S_{n-1}-P$ state during $\tau_{\text {off }}$. To derive the single-molecule kinetics for a single nanoparticle, the concentrations in Eq. (5) need to be replaced by the probabilities $P(t)$ of finding the nanoparticle in the states $\mathrm{Au}_{m} \mathrm{~S}_{n}$ and $\mathrm{Au}_{m} \mathrm{~S}_{n-1}-\mathrm{P}$ at time $t$. Then, Eq. (5) becomes

$$
\frac{\mathrm{d} P_{\mathrm{Au}_{m} \mathrm{~S}_{n-1} \mathrm{P}}(t)}{\mathrm{d} t}=-\frac{\mathrm{d} P_{\mathrm{Au}_{m} \mathrm{~S}_{n}}(t)}{\mathrm{d} t}=\gamma_{\mathrm{app}} P_{\mathrm{Au}_{m} S_{n}}(t)
$$

where $P_{\mathrm{Au}_{m} \mathrm{~s}_{n}}(t)+P_{\mathrm{Au}_{m} \mathrm{~s}_{n-1}-\mathrm{P}}(t)=1$. At the onset of each 
$\tau_{\text {off }}$ (i.e., $t=0$ ), no product molecule has formed. So the initial conditions for solving Eq. (6) are $P_{\mathrm{Au}_{m} \mathrm{~S}_{n}}(0)=1$ and $P_{\mathrm{Au}_{m} \mathrm{~S}_{n-1}-\mathrm{P}}(0)=0$. In single-nanoparticle experiments, the depletion of substrate is negligible during catalysis and [S] is time-independent; it is therefore valid to treat $\gamma_{\text {app }}$ as a pseudo-first-order rate constant.

We can then evaluate the probability density of the time $\tau$ required to complete the $\tau_{\text {off }}$ reaction, $f_{\text {off }}(\tau)$, i.e., the probability density of $\tau_{\text {off }}$ The probability for finding a particular $\tau$ is $\left.f_{\text {off }} \tau\right) \Delta \tau$, which is equal to the probability of switching from the $\mathrm{Au}_{m} \mathrm{~S}_{n}$ state to the $\mathrm{Au}_{m} \mathrm{~S}_{n-1}-\mathrm{P}$ state for the nanoparticle between $t=\tau$ and $\tau+\Delta \tau$, which is $\Delta P_{\mathrm{Au}_{m} \mathrm{~s}_{n-1}-\mathrm{P}}(\tau)=\gamma_{\mathrm{app}} P_{\mathrm{Au}_{m} \mathrm{~s}_{n}}(\tau) \Delta \tau$. In the limit of infinitesimal $\Delta \tau$,

$$
\begin{aligned}
f_{\text {off }}(\tau) & =\frac{\mathrm{d} P_{\mathrm{Au}_{m} \mathrm{~S}_{n-1}-\mathrm{P}}(\tau)}{\mathrm{d} \tau}=\gamma_{\mathrm{app}} P_{\mathrm{Au}_{m} \mathrm{~S}_{n}}(\tau) \\
& =\frac{\gamma_{\mathrm{eff} K_{1}[\mathrm{~S}]}}{1+K_{1}[\mathrm{~S}]} P_{\mathrm{Au}_{m} S_{n}}(\tau)
\end{aligned}
$$

Solving Eq. (6) for $P_{\mathrm{Au}_{m} \mathrm{~S}_{n}}(\tau)$ with the initial conditions, we obtain

$$
f_{\text {off }}(\tau)=\frac{\gamma_{\text {eff }} K_{1}[S]}{1+K_{1}[S]} \exp \left(-\frac{\gamma_{\text {eff }} K_{1}[S]}{1+K_{1}[S]} \tau\right)
$$

Clearly, regardless of the values of $\gamma_{\text {eff }}\left(=k n_{\mathrm{T}}\right)$ and $K_{1}, f_{\text {off }}(\tau)$ is a single-exponential decay function with the [S]-dependent decay constant $\gamma_{\text {eff }} K_{1}[S] /\left(1+K_{1}[S]\right)$. At saturating substrate concentrations where all surface catalytic sites are occupied by substrates, $\theta_{S}=1$ and $f_{\text {off }}(\tau)=\gamma_{\text {eff }} \exp \left(-\gamma_{\text {eff }} \tau\right)$. Figure 3(a) shows a typical experimental histogram of $\tau_{\text {off }}$ for a single 6-nm Au-nanoparticle at a saturating [S]; the exponential distribution is clear; fitting the distribution gives $\gamma_{\text {eff }}=0.33 \mathrm{~s}^{-1} \pm 0.02 \mathrm{~s}^{-1}$, which quantifies the catalytic reactivity of this Au-nanoparticle [17]. By determining $\gamma_{\text {eff }}$ for every nanoparticle from its distribution of $\tau_{\text {off }}$ we can obtain the distribution of $\gamma_{\text {eff }}$ among many nanoparticles (Fig. 3(a), inset). The broad distribution of $\gamma_{\text {eff }}$ quantifies the heterogeneity in catalytic reactivity of the 6-nm Au-nanoparticles, which is unavailable from conventional ensemble measurements of nanoparticle catalysis.

The first moment of $f_{\text {off }}(\tau),\left\langle\tau_{\text {off }}\right\rangle=\int_{0}^{\infty} \tau f_{\text {off }}(\tau) \mathrm{d} \tau$, gives the mean waiting time $\tau_{\text {off }}$ for completing the catalytic product formation reaction; $\left\langle\tau_{\text {off }}\right\rangle^{-1}$ then represents the rate of product formation for a single nanoparticle:

$$
\left\langle\tau_{\text {off }}\right\rangle^{-1}=\frac{\gamma_{\text {eff }} K_{1}[\mathrm{~S}]}{1+K_{1}[\mathrm{~S}]}
$$

This equation resembles the classic LangmuirHinshelwood rate equation [26]; we thus call it the single-molecule Langmuir-Hinshelwood equation. This equation predicts a hyperbolic dependence of $\left\langle\tau_{\text {off }}\right\rangle^{-1}$ on the substrate concentration with a saturation value of $\gamma_{\text {eff }}$ at high substrate concentrations.

To give a physical interpretation of Eq. (9): the maximum product formation rate is reached when all surface catalytic sites are occupied by substrates, and the reaction rate $\left\langle\tau_{\text {off }}\right\rangle^{-1}$ equals the reactivity per catalytic site $(k)$ multiplied by the total number $\left(n_{\mathrm{T}}\right)$ of surface catalytic sites, i.e., $\gamma_{\text {eff }}$. Figure $3(b)$ shows the experimental data of 6-nm Au-nanoparticles, where each data point is averaged over many particles; the

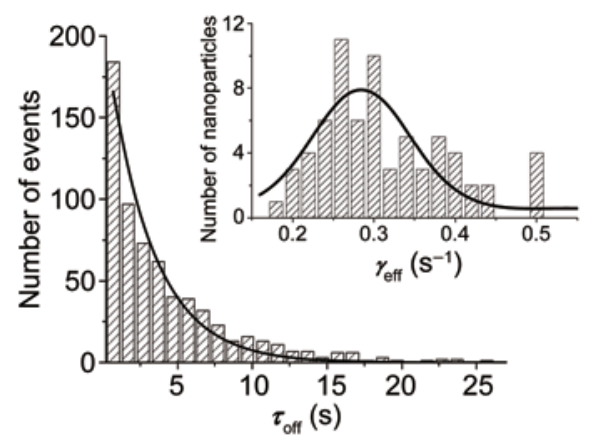

(a)

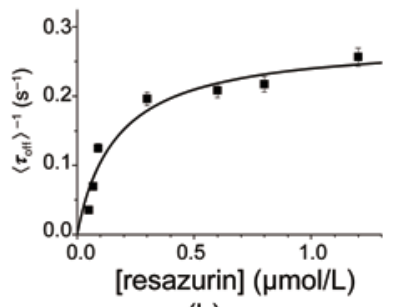

(b)

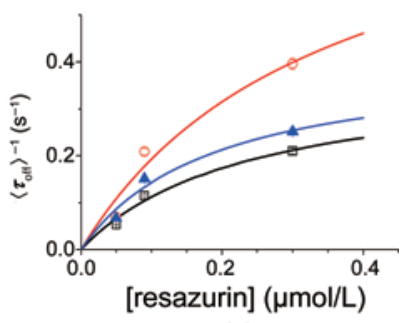

(c)
Figure 3 (a) Distribution of $\tau_{\text {off }}$ from the fluorescence turnover trajectory of a single 6-nm Au-nanoparticle at a saturating substrate concentration. The solid line is a single-exponential fit with $\gamma_{\text {eff }}=$ $0.33 \mathrm{~s}^{-1}$. Inset: distribution of $\gamma_{\text {eff }}$ among many Au-nanoparticles; the solid line is a Gaussian fit with center at $0.28 \mathrm{~s}^{-1}$ and full-widthat-half-maximum (FWHM) of $0.12 \mathrm{~s}^{-1}$. (b) Resazurin concentration dependence of $\left\langle\tau_{\text {off }}\right\rangle^{-1}$ averaged over many Au-nanoparticles. The solid line is a fit with Eq. (9) with $\gamma_{\text {eff }}=0.28 \mathrm{~s}^{-1}, K_{1}=6(\mu \mathrm{mol} / \mathrm{L})^{-1}$. (c) Resazurin concentration dependence of $\left\langle\tau_{\text {off }}\right\rangle^{-1}$ from three 6-nm Au-nanoparticles. Each titration is from one nanoparticle. Solid lines are fits with Eq. (9). Figures adapted from Xu et al. [15, 17] 
saturation behavior of $\left\langle\tau_{\text {off }}\right\rangle^{-1}$ is clear [15].

On the other hand, if we consider at the singleparticle level, Eq. (9) predicts $\left\langle\tau_{\text {off }}\right\rangle^{-1}$ to show variable saturation levels and initial slopes with increasing [S], if different Au-nanoparticles have heterogeneous catalytic reactivity $\left(\gamma_{\text {eff }}\right)$ and substrate binding affinity $\left(K_{1}\right)$. This is indeed observed in the experimental [S] titration curves of $\left\langle\tau_{\text {off }}\right\rangle^{-1}$ of individual Aunanoparticles (Fig. 3(c)) [15]. These variable behaviors are further manifestations of the heterogeneous catalytic reactivity among the individual Aunanoparticles, which are usually hidden in ensemble measurements.

\section{$2.2 \tau_{\text {on }}$ reaction: two-pathway mechanism for product dissociation}

In a single-particle turnover trajectory, $\tau_{\text {on }}$ starts at the moment when a product molecule forms on a particle surface, and ends once this product leaves the particle surface. Based on the two-pathway mechanism for product dissociation in Fig. 2, the processes occurring in $\tau_{\text {on }}$ are either reactions (ii) and (iii):

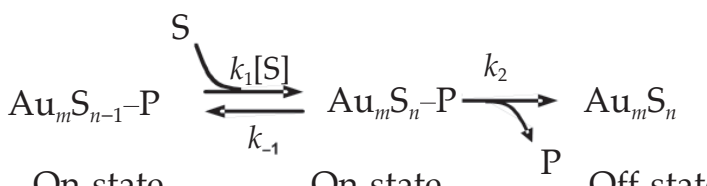

On-state On-state Off-state

or reaction (iv):

$$
\begin{array}{ccc}
\mathrm{Au}_{m} \mathrm{~S}_{n-1}-\mathrm{P} & \stackrel{k_{3}}{\mathrm{P}} & \mathrm{Au}_{m} \mathrm{~S}_{n-1} \\
\text { On-state } & & \text { Off-state }
\end{array}
$$

Under the assumptions of the LangmuirHinshelwood mechanism where fast substrate adsorption equilibrium is established at all times, the $\mathrm{Au}_{m} \mathrm{~S}_{n-1}$ state will be immediately converted to the $\mathrm{Au}_{m} \mathrm{~S}_{n}$ state, as substrate molecules in the solution will quickly bind to an available site to maintain the equilibrium. The corresponding single-molecule rate equations for a single particle are

$$
\begin{aligned}
& \frac{\mathrm{d} P_{\mathrm{Au}_{m} \mathrm{~S}_{n-1}-\mathrm{P}}(t)}{\mathrm{d} t}=-\left(k_{1}[\mathrm{~S}]+k_{3}\right) P_{\mathrm{Au}_{m} \mathrm{~S}_{n-1}-\mathrm{P}}(t)+k_{-1} P_{\mathrm{Au}_{m} \mathrm{~S}_{n}-\mathrm{P}}(t) \\
& \frac{\mathrm{d} P_{\mathrm{Au}_{m} \mathrm{~S}_{n}-\mathrm{P}}(t)}{\mathrm{d} t}=k_{1}[\mathrm{~S}] P_{\mathrm{Au}_{m} \mathrm{~S}_{n-1}-\mathrm{P}}(t)-\left(k_{2}+k_{-1}\right) P_{\mathrm{Au}_{m} \mathrm{~S}_{n}-\mathrm{P}}(t)
\end{aligned}
$$

$$
\begin{aligned}
& \frac{\mathrm{d} P_{\mathrm{Au}_{m} \mathrm{~s}_{n}}(t)}{\mathrm{d} t}=k_{2} P_{\mathrm{Au}_{m} \mathrm{~S}_{n}-\mathrm{P}}(t) \\
& \frac{\mathrm{d} P_{\mathrm{Au}_{m} \mathrm{~S}_{\mathrm{n}-1}}(t)}{\mathrm{d} t}=k_{3} P_{\mathrm{Au}_{m} \mathrm{~S}_{n-1}-\mathrm{P}}(t)
\end{aligned}
$$

with the initial conditions $P_{\mathrm{Au}_{m} \mathrm{~S}_{n-1}-\mathrm{P}}(0)=1$ and $P_{\mathrm{Au}_{m} \mathrm{~S}_{n}-\mathrm{P}}(0)=P_{\mathrm{Au}_{m} \mathrm{~S}_{n}}(0)=P_{\mathrm{Au}_{m} \mathrm{~S}_{n-1}}(0)=0$. At any time within $\tau_{\text {on }} P_{\mathrm{Au}_{m} \mathrm{~S}_{n-1}-\mathrm{P}}(t)+P_{\mathrm{Au}_{m} \mathrm{~s}_{n}-\mathrm{P}}(t)+P_{\mathrm{Au}_{m} \mathrm{~S}_{n}}(0)+P_{\mathrm{Au}_{m} \mathrm{~s}_{n-1}}(t)=1$.

We can then consider the probability density $f_{\text {on }}(\tau)$ of the $\tau_{\text {on }}$. $\tau_{\text {on }}$ is the time required to finish reactions in Eq. (10a), or the time required to finish the reaction in Eq. (10b). The probability of finding a particular $\tau$ is $f_{\text {on }}(\tau) \Delta \tau$, which is equal to the sum of (1) the probability of switching from the $\mathrm{Au}_{m} \mathrm{~S}_{n}-\mathrm{P}$ state to the $\mathrm{Au}_{m} \mathrm{~S}_{n}$ state between the time $\tau$ and $\tau+\Delta \tau$ and (2) the probability of switching from the $\mathrm{Au}_{m} \mathrm{~S}_{n-1}-\mathrm{P}$ state to the $\mathrm{Au}_{m} \mathrm{~S}_{n-1}$ state between the time $\tau$ and $\tau+$ $\Delta \tau$. The first probability is $\Delta P_{\mathrm{Au}_{m} \mathrm{~S}_{n}}(\tau)$, which equals $k_{2} P_{\mathrm{Au}_{m} \mathrm{~S}_{n}-\mathrm{P}}(\tau) \Delta \tau$. The second probability is $\Delta P_{\mathrm{Au}_{m} \mathrm{~S}_{n-1}}(\tau)$, which equals $k_{3} P_{\mathrm{Au}_{m} \mathrm{~s}_{n-1}-\mathrm{P}}(\tau) \Delta \tau$. Then $f_{\text {on }}(\tau)$ is

$$
\begin{aligned}
f_{\text {on }}(\tau) & =\left.\frac{\mathrm{d} P_{\mathrm{Au}_{m} \mathrm{~S}_{n}}(t)}{\mathrm{d} t}\right|_{t=\tau}+\left.\frac{\mathrm{d} P_{\mathrm{Au}_{m} \mathrm{~S}_{n-1}}(t)}{\mathrm{d} t}\right|_{t=\tau} \\
& =k_{2} P_{\mathrm{Au}_{m} \mathrm{~S}_{n}-\mathrm{P}}(\tau)+k_{3} P_{\mathrm{Au}_{m} \mathrm{~S}_{n-1}-\mathrm{P}}(\tau)
\end{aligned}
$$

Solving Eqs. (11a)-(11d) for $P_{\mathrm{Au}_{m} \mathrm{~S}_{n}-\mathrm{P}}(\tau)$ and $P_{\mathrm{Au}_{m} \mathrm{~S}_{n-1}-\mathrm{P}}(\tau)$ by Laplace transform with the initial conditions, we can get:

$$
f_{\text {on }}(\tau)=\frac{1}{2 \alpha}\left[M \mathrm{e}^{(\beta+\alpha) \tau}+N \mathrm{e}^{(\beta-\alpha) \tau}\right]
$$

where $\alpha=\sqrt{\left(k_{1}[\mathrm{~S}]+k_{-1}+k_{2}+k_{3}\right)^{2} / 4-\left(k_{1} k_{2}[\mathrm{~S}]+k_{-1} k_{3}+k_{2} k_{3}\right)}$, $\beta=-\left(k_{1}[\mathrm{~S}]+k_{-1}+k_{2}+k_{3}\right) / 2, M=k_{2} k_{1}[\mathrm{~S}]+k_{3} \alpha+k_{3} \beta+k_{3} k_{-1}+k_{3} k_{2}$ ， and $N=-k_{2} k_{1}[\mathrm{~S}]+k_{3} \alpha-k_{3} \beta-k_{3} k_{-1}-k_{3} k_{2}$. At high substrate concentrations, Eq. (13) reduces to a singleexponential decay function, $f_{\text {on }}(\tau)_{[\mathrm{S}] \rightarrow \infty} \approx k_{2} \mathrm{e}^{-k_{2} \tau}$, with $k_{2}$, the product dissociation rate constant in the substrate-assisted pathway, being the decay rate constant (Fig. 2). Figure 4(a) shows the experimental histogram of $\tau_{\text {on }}$ from the turnover trajectory of a single 6-nm Au-nanoparticle at a saturating substrate concentration; fitting it with a single-exponential decay function gives $k_{2}$ for this nanoparticle directly [17]. By determining $k_{2}$ for every nanoparticle from 


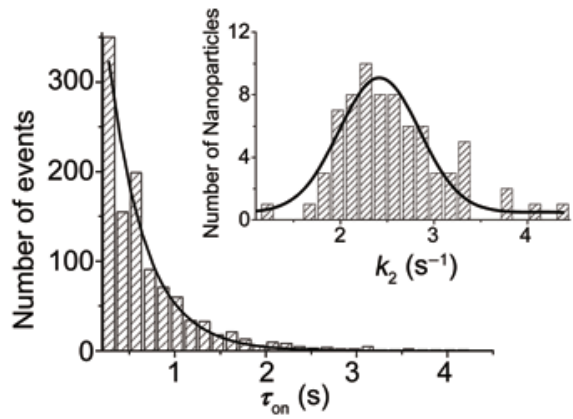

(a)

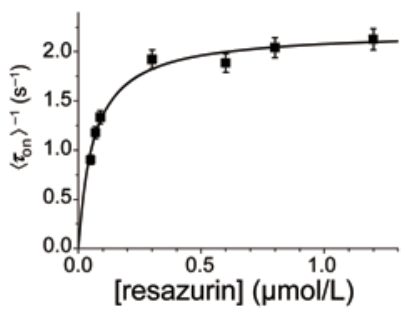

(b)

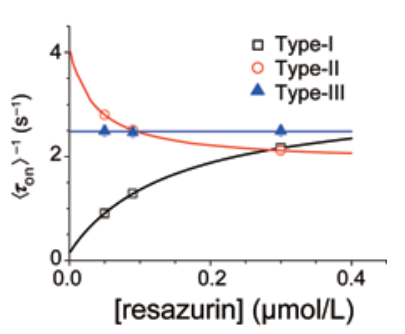

(c)
Figure 4 (a) Distribution of $\tau_{\text {on }}$ from a fluorescence turnover trajectory of a 6-nm Au-nanoparticle at a saturating substrate concentration. The solid line is a single-exponential fit with $k_{2}=2.5$ $\mathrm{s}^{-1}$. Inset: distribution of $k_{2}$; the solid line is a Gaussian fit centered at $2.4 \mathrm{~s}^{-1}$ with FWHM of $0.9 \mathrm{~s}^{-1}$. (b) Resazurin concentration dependence of $\left\langle\tau_{\text {on }}\right\rangle^{-1}$ averaged over many Au-nanoparticles. The solid line is a fit with Eq. (14) with $k_{2}=2.2 \mathrm{~s}^{-1}, K_{2}=16(\mu \mathrm{mol} / \mathrm{L})^{-1}$, and $k_{3}$ $=0 \mathrm{~s}^{-1}$. (c) Resazurin concentration dependence of $\left\langle\tau_{\text {on }}\right\rangle^{-1}$ from three individual Au-nanoparticles with Type I, Type II, and Type III behaviors. The solid lines are fits with Eq. (14): for the Type I nanoparticle, $k_{2}=3.2 \mathrm{~s}^{-1}, K_{2}=6.7(\mu \mathrm{mol} / \mathrm{L})^{-1}$, and $k_{3}=0.15 \mathrm{~s}^{-1}$; for the Type II, $k_{2}=1.8 \mathrm{~s}^{-1}, K_{2}=28(\mu \mathrm{mol} / \mathrm{L})^{-1}$, and $k_{3}=4.1 \mathrm{~s}^{-1}$; for the Type III, $k_{2}=k_{3}=2.4 \mathrm{~s}^{-1}$ (or $k_{2}=0$ ), and $K_{2}=$ arbitrary value. Figures adapted from $\mathrm{Xu}$ et al. $[15,17]$

its distribution of $\tau_{\text {on }}$, we can get the distribution of $k_{2}$ among nanoparticles (Fig. 4(a), inset). The broad distribution of $k_{2}$ reflects the activity heterogeneity in the product dissociation reaction among the nanoparticles.

From $f_{\text {on }}(\tau)$, we can derive $\left\langle\tau_{\text {on }}\right\rangle^{-1}$, the timeaveraged product dissociation rate for a single nanoparticle,

$$
\left\langle\tau_{\text {on }}\right\rangle^{-1}=1 / \int_{0}^{\infty} \tau f_{\text {on }}(\tau) \mathrm{d} \tau=\frac{k_{2} K_{2}[\mathrm{~S}]+k_{3}}{1+K_{2}[\mathrm{~S}]}
$$

where $K_{2}=k_{1} /\left(k_{-1}+k_{2}\right)$. At the limiting condition of [S] $\rightarrow 0,\left\langle\tau_{\text {on }}\right\rangle^{-1}=k_{3}$, which is the rate constant for direct product dissociation. This is because the forward reaction of reaction (ii) in Fig. 2 is negligible $\left(k_{1}[\mathrm{~S}]\right.$ $=0)$ and the direct product dissociation dominates. At the limiting condition of saturating substrate concentrations (i.e., [S] $\rightarrow \infty$ ), $\left\langle\tau_{\text {on }}\right\rangle^{-1}=k_{2}$, which is the product dissociation rate constant in the substrateassisted pathway. This is because the $\mathrm{Au}_{m} \mathrm{~S}_{n-1}-\mathrm{P}$ state will be immediately converted to the $\mathrm{Au}_{m} \mathrm{~S}_{n}-\mathrm{P}$ state via reaction (ii) due to large [S]; then the product dissociation dominantly takes the substrate-assisted pathway and the reaction rate is determined by $k_{2}$.

With different relative magnitudes of $k_{2}$ and $k_{3}$, Eq. (14) immediately predicts three types of [S] dependence of $\left\langle\tau_{\text {on }}\right\rangle^{-1}$ : Type I : $\left\langle\tau_{\text {on }}\right\rangle^{-1}$ increases with increasing [S] and eventually saturates if $k_{2}>$ $k_{3}$; Type II : $\left\langle\tau_{\text {on }}\right\rangle^{-1}$ decreases with increasing [S] and flattens if $k_{2}<k_{3}$; Type III: $\left\langle\tau_{\text {on }}\right\rangle^{-1}$ is independent of [S] if $k_{2}=k_{3}$, or $K_{2}=0$. All three types of behaviors are observed for individual 6-nm Au-nanoparticles (Fig. 4(c)), and they have different subpopulations: $66 \%$ of Au-nanoparticles are Type I , 19\% are Type II , and $15 \%$ are Type III[15]. These different behaviors are manifestations of the differential selectivity of individual Au-nanoparticles between the two parallel product dissociation pathways. This differential selectivity is completely hidden in nanoparticleaveraged results, which are dominated by the behavior of the Type I particles (Fig. 4(b)).

\subsection{Overall rate of turnovers}

From the fluorescence turnover trajectory, the overall rate of turnovers for a single particle can be determined by counting the number of off-on events per unit time, $\left\langle\tau_{\text {off }+ \text { on }}\right\rangle^{-1}$. From Eqs. (9) and (14), we get:

$$
\begin{gathered}
\left\langle\tau_{\text {off }+ \text { on }}\right\rangle^{-1}=\left\langle\tau_{\text {off }}+\tau_{\text {on }}\right\rangle^{-1}=\left(\left\langle\tau_{\text {off }}\right\rangle+\left\langle\tau_{\text {on }}\right\rangle\right)^{-1} \\
=\frac{\gamma_{\text {eff }} k_{2} K_{1} K_{2}[\mathrm{~S}]^{2}+\gamma_{\text {eff }} k_{3} K_{1}[\mathrm{~S}]}{\left(\gamma_{\text {eff }}+k_{2}\right) K_{1} K_{2}[\mathrm{~S}]^{2}+\left(\gamma_{\text {eff }} K_{1}+k_{3} K_{1}+k_{2} K_{2}\right)[\mathrm{S}]+k_{3}}
\end{gathered}
$$

As expected, the rate of the turnovers contains kinetic parameters for both the catalytic product formation reaction $\left(\gamma_{\text {eff }}\right.$ and $\left.K_{1}\right)$ and the product dissociation reaction $\left(k_{2}, k_{3}\right.$, and $\left.K_{2}\right)$. At $[\mathrm{S}]=0,\left\langle\tau_{\text {off }+ \text { on }}\right\rangle_{[\mathrm{S}]=0}^{-1}=0$ and no catalysis occurs; at $[S] \rightarrow \infty,\left\langle\tau_{\text {off } 0 \text { on }}\right\rangle_{[\mathrm{S}] \rightarrow \infty}^{-1}=\gamma_{\text {eff }}$. $k_{2} /\left(\gamma_{\text {eff }}+k_{2}\right)$, which reduces to $\gamma_{\text {eff }}$ when the catalytic conversion reaction is rate-limiting in the catalytic cycle (i.e., $y_{\text {eff }}<<k_{2}$ ).

As compared with those of $\left\langle\tau_{\text {off }}\right\rangle^{-1}$ and $\left\langle\tau_{\text {on }}\right\rangle^{-1}$ (Eqs. (9) and (14)), the much more complicated expression 
of $\left\langle\tau_{\text {off }+ \text { on }}\right\rangle^{-1}$ highlights the distinct advantage of single-molecule detection of nanoparticle catalysis, in which each catalytic turnover is temporally resolved into the $\tau_{\text {off }}$ and $\tau_{\text {on }}$ parts. Figure 5(a) shows the [S] dependence of $\left\langle\tau_{\text {off }+ \text { on }}\right\rangle^{-1}$ from 6-nm Au-nanoparticles. The [S]-dependent saturation kinetics of $\left\langle\tau_{\text {off }+ \text { on }}\right\rangle^{-1}$ is clear, as well as expected because of the catalytic product formation reaction. The [S]-dependent kinetics of the product dissociation reaction cannot be identified in $\left\langle\tau_{\text {off }+ \text { on }}\right\rangle^{-1}$, however, without separately examining the [S] dependence of $\left\langle\tau_{\text {on }}\right\rangle^{-1}$ (Figs. 4(b) and $4(\mathrm{c}))$.

As a comparison, Fig. 5(b) shows the bulk [S] dependence of the initial reaction rate (i.e., resazurin consumption rate) in the presence of a large excess of the co-reactant $\mathrm{NH}_{2} \mathrm{OH}$. The reaction rate is determined by monitoring the absorption band of the substrate resazurin using UV-Vis spectrophotometry. The initial reaction rate shows saturation kinetics with increasing [S] and can be fitted by the conventional ensemble Langmuir-Hinshelwood equation, rate $=k_{\text {eff }} K[\mathrm{AuNP}][\mathrm{S}] /(1+K[S])$, where $k_{\text {eff }}$ is the catalytic conversion rate constant per particle, $K$ is the substrate adsorption equilibrium constant, and [AuNP] is the concentration of the Au-nanoparticles. This equation is for unimolecular reactions, to which our reaction approximates in the presence of excess $\mathrm{NH}_{2} \mathrm{OH}$, and it also assumes fast product desorption relative to the catalytic conversion rate. Fitting the bulk kinetic data gives $k_{\text {eff }}=0.63 \mathrm{~s}^{-1} \pm 0.03 \mathrm{~s}^{-1}$ and $K$ $=0.29(\mu \mathrm{mol} / \mathrm{L})^{-1} \pm 0.04(\mu \mathrm{mol} / \mathrm{L})^{-1}$, comparable to $\gamma_{\text {eff }}\left(\sim 0.28 \mathrm{~s}^{-1}\right)$ and $K_{1}\left(\sim 6(\mu \mathrm{mol} / \mathrm{L})^{-1}\right)$ obtained from the nanoparticle-averaged single-molecule kinetics measurements (Fig. 3(b)). The differences between the bulk kinetics and the single-molecule kinetics could arise from the differences in experimental conditions - the bulk kinetics refers to nanoparticles free in solution, while the single-molecule kinetics refers to those immobilized on amine-functionalized glass surfaces.

Clearly, the bulk kinetic measurements in Fig. 5(b) can neither separate the kinetics of catalytic conversion and that of product dissociation, nor reveal the [S]-dependent kinetics of the product dissociation reaction, nor determine the activity variations among individual nanoparticles. In contrast, the single-molecule measurements make it possible to interrogate the behaviors of individual nanoparticles, and for each nanoparticle, to separate its catalytic kinetics into two temporal parts.

\section{Single-molecule Langmuir-Hinshelwood kinetics for catalysis of oligomeric enzymes}

The Langmuir-Hinshelwood mechanism explicitly includes the multiplicity of catalytic sites. Our single-

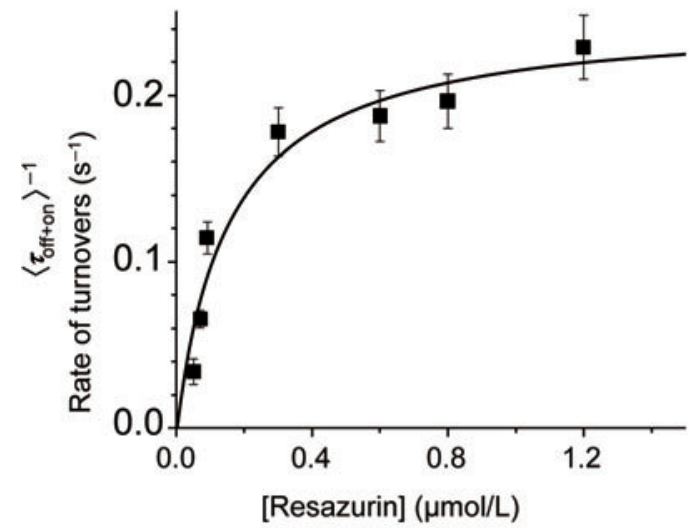

(a)

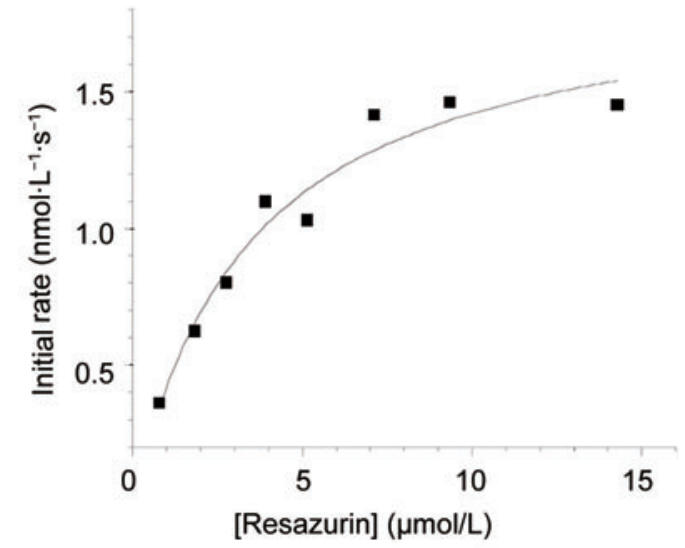

(b)

Figure 5 (a) Resazurin concentration dependence of the rate of turnovers, $\left\langle\tau_{\text {offton }}\right\rangle^{-1}$. Each data point is calculated from the values of $\tau_{\text {off }}$ and $\tau_{\text {on }}$ of many single-particle fluorescence trajectories. The solid line is a plot of Eq. (15) using kinetic parameters from Figs. 3(b) and 4(b). Figure adapted from Xu et al. [15]. (b) Bulk measurements of resazurin concentration dependence of initial reaction rate in the presence of a large excess of $\mathrm{NH}_{2} \mathrm{OH}$. Au nanoparticle concentration $[\mathrm{AuNP}]=3 \mathrm{nmol} / \mathrm{L},\left[\mathrm{NH}_{2} \mathrm{OH}\right]=1 \mathrm{mmol} / \mathrm{L}$. The solid line is a fit with the conventional LangmuirHinshelwood equation, rate $=k_{\text {eff }} K\left[\right.$ AuNP][S]/(1+K[S]) with $k_{\text {eff }}=0.63 \mathrm{~s}^{-1} \pm 0.03 \mathrm{~s}^{-1}$, and $K=0.29(\mu \mathrm{mol} / \mathrm{L})^{-1} \pm 0.04(\mu \mathrm{mol} / \mathrm{L})^{-1}$ 
molecule kinetic formulation of this mechanism enables it to be used to treat the catalysis of nanoparticle catalysts at the single-molecule level. This single-molecule Langmuir-Hinshelwood kinetics can also be applied to the catalysis of enzymes, particularly to oligomeric enzymes that have multiple active sites [16].

For enzyme catalysis, the most common mechanism is the classic Michaelis-Menten mechanism. Single-molecule kinetic theory has been formulated for this mechanism [27-40] and applied to analyze the results of many single-enzyme studies [19-22, 28, 41-47]. Although powerful, the classic Michaelis-Menten mechanism is a one-site, onesubstrate kinetic model. Yet, many enzymes are oligomeric with multiple catalytic sites, such as the tetrameric enzyme $\beta$-galactosidase that has been studied at the single-molecule level [21, 44, 48]. Including the multiplicity of catalytic sites in the single-molecule kinetic formulism is thus desirable; the Langmuir-Hinshelwood mechanism provides an option [16].

In order to apply the Langmuir-Hinshelwood mechanism to oligomeric enzymes, a prerequisite is that substrate binding/unbinding must be significantly faster than the catalytic conversion reaction, so an equilibrium occupation of all active sites is established before a catalytic conversion occurs at one of the sites. An example that satisfies this prerequisite is the tetrameric enzyme $\beta$-galactosidase [21, 44, 48]. We can then use the formulism of Eq. (2) of the Langmuir-Hinshelwood mechanism:

$$
\gamma_{\text {app }}=k_{\text {cat }} n
$$

where $\gamma_{\text {app }}$ is the apparent rate constant for an oligomeric enzyme to form one product at one of its active sites, $k_{\text {cat }}$ is the single-site rate constant for catalytic conversion, and $n$ is the number of substrate molecules bound by the enzyme at equilibrium. Since the total number $\left(n_{\mathrm{T}}\right)$ of active sites of oligomeric enzymes is typically small, the relative fluctuation of the number of bound substrates can be large from one instant to another; but, the equilibrium occupation $n$ is always described by its equilibrium binding isotherm and can be fractional at certain substrate concentrations. If the site-site interactions are insignificant among the monomers of the oligomeric enzyme, i.e., monomers are independent of each other for substrate binding, $n$ obeys the Langmuir isotherm: $n=n_{\mathrm{T}} K[S] /(1+K[S])$, where $K$ is the binding equilibrium constant. Expressions similar to Eqs. (8) and (9) can then be obtained to describe $f_{\text {off }}(\tau)$ and $\left\langle\tau_{\text {off }}\right\rangle{ }^{-1}$ for oligomeric enzymes. If the site-site interactions are significant and there is cooperativity for substrate binding to the multiple active sites, alternative binding isotherms can then be used to describe $n$ at different substrate concentrations. For example, if positive cooperativity exists for substrate binding, we can use a sigmoidal isotherm, such as the Monod-Wyman-Changeux model developed for the cooperative $\mathrm{O}_{2}$ binding to hemoglobin [49], to substitute the Langmuir isotherm to formulate the single-molecule kinetic equations.

The single-molecule Langmuir-Hinshelwood formulism for oligomeric enzymes can be used to treat the results of recent single-molecule enzyme studies. These studies have revealed large activity heterogeneity from molecule to molecule and, for a single molecule, from time to time $[20,21,28,42$, $50,51]$. Most of them attributed the heterogeneity in activity to variability in $k_{\text {cat }}$ caused by different enzyme conformations. The inclusion of the number of catalytic sites in the single-molecule kinetic equations offers an additional opportunity to evaluate possible causes of the activity heterogeneity of oligomeric enzymes, for example, differences in the number of available active sites in an oligomeric enzyme that can result from allosteric monomer-monomer interactions or from enzyme conformational dynamics.

\section{Concluding remarks}

We have discussed the single-molecule kinetic analysis of the fluorescence turnover trajectories of individual nanoparticle catalysts. A kinetic mechanism can be formulated, and quantitative kinetic information can be extracted at the singleparticle level, which enables the quantification of reactivity heterogeneity and reveals the differential selectivity between parallel reaction pathways for 
individual nanoparticles. Because of its explicit inclusion of the multiplicity of catalytic sites, the single-molecule Langmuir-Hinshelwood formulism has an advantage in treating the single-molecule kinetics of oligomeric enzymes that contain multiple catalytic sites.

As the Langmuir-Hinshelwood mechanism is general for many heterogeneous catalysts [26], our formulism should provide a general theoretical framework to understand single-molecule kinetics of heterogeneous catalysis. Still, more work remains to be done, for example, to include the substrate binding/ unbinding kinetics explicitly without assuming a fast substrate adsorption equilibrium. One way is to assume one "effective" site per particle and use a modified Michaelis-Menten mechanism, as we showed in our previous paper [16]. Although it misses the multiplicity of the surface active sites, this modified Michaelis-Menten mechanism does include substrate binding and unbinding explicitly.

Another issue is the variability of surface sites on a single particle-there are different types of sites on the particle surface, for example sites at the corners, edges, or facets. These sites are expected to have different activity. Can we include the differences between the various sites on a single particle in the theory? This also represents an experimental challenge: can we differentiate reactions occurring at different sites on a single particle?

Furthermore, the single-particle turnover trajectories contain time-dependent information about the catalytic kinetics at the single-turnover resolution. Our experimental studies have revealed large temporal activity fluctuations of individual Au-nanoparticles, which are attributable to both catalysis-induced and spontaneous dynamic surface restructuring $[15,17]$. Inclusion of these temporal catalytic dynamics in the single-molecule kinetic formulation should offer more insights into the fundamental catalytic properties of nanoparticle catalysts.

\section{Acknowledgments}

We thank the Army Research Office (56355-CH), National Science Foundation (NSF, No. CBET-
0851257), American Chemical Society Petroleum Research Foundation (No. 47918-G5), and NSFfunded Cornell Center for Materials Research for financial support.

\section{References}

[1] Somorjai, G. A.; Contreras, A. M.; Montano, M.; Rioux, R. M. Clusters, surfaces, and catalysis. Proc. Natl. Acad. Sci. USA 2006, 103, 10577-10583.

[2] Bell, A. T. The impact of nanoscience on heterogeneous catalysis. Science 2003, 299, 1688-1691.

[3] Burda, C.; Chen, X. B.; Narayanan, R.; El-Sayed, M. A. Chemistry and properties of nanocrystals of different shapes. Chem. Rev. 2005, 105, 1025-1102.

[4] Heiz, U.; Landman, U. Nanocatalysis; Springer: Berlin, 2007.

[5] Xia, Y. N.; Xiong, Y. J.; Lim, B.; Skrabalak, S. E. Shapecontrolled synthesis of metal nanoparticles: Simple chemistry meets complex physics? Angew. Chem. Int Ed. 2008, 48, 60-103.

[6] Tao, A. R.; Habas, S.; Yang, P. D. Shape control of colloidal metal nanocrystals. Small 2008, 4, 310-325.

[7] Grzelczak, M.; Pérez-Juste, J.; Mulvaney, P.; Liz-Marzán, L. M. Shape control in gold nanoparticle synthesis. Chem. Soc. Rev. 2008, 37, 1783-1791.

[8] Fan, F. -R. F.; Kwak, J.; Bard, A. J. Single molecule electrochemistry. J. Am. Chem. Soc. 1996, 118, 96699675.

[9] Fan, F. -R. F.; Bard, A. J. An electrochemical Coulomb staircase: Detection of single electron-transfer events at nanometer electrodes. Science 1997, 277, 1791-1793.

[10] Meier, J.; Friedrich, K. A.; Stimming, U. Novel method for the investigation of single nanoparticle reactivity. Faraday Discuss. 2002, 121, 365-372.

[11] Meier, J.; Schiotz, J.; Liu, P.; Norskov, J. K.; Stimming, U. Nano-scale effects in electrochemistry. Chem. Phys. Lett. 2004, 390, 440-444.

[12] Chen, S. L.; Kucernak, A. Electrocatalysis under conditions of high mass transport: Investigation of hydrogen oxidation on single submicron Pt particles supported on carbon. J. Phys. Chem. B 2004, 108, 13984-13994.

[13] Krapf, D.; Wu, M. -Y.; Smeets, R. M. M.; Zandbergen, H. W.; Dekker, C.; Lemay, S. G. Fabrication and 
characterization of nanopore-based electrodes with radii down to $2 \mathrm{~nm}$. Nano Lett. 2006, 6, 105-109.

[14] Novo, C.; Funston, A. M.; Mulvaney, P. Direct observation of chemical reactions on single gold nanocrystals using surface plasmon spectroscopy. Nat. Nanotechnol. 2008, 3, 598-602.

[15] Xu, W.; Kong, J. S.; Yeh, Y. -T. E.; Chen, P. Singlemolecule nanocatalysis reveals heterogeneous reaction pathways and catalytic dynamics. Nat. Mater. 2008, 7, 992-996.

[16] Xu, W.; Kong, J. S.; Chen, P. Single-molecule kinetic theory of heterogeneous and enzyme catalysis. J. Phys. Chem. C 2009, 113, 2393-2404.

[17] Xu, W.; Kong, J. S.; Chen, P. Probing the catalytic activity and heterogeneity of Au-nanoparticles at the singlemolecule level. Phys. Chem. Chem. Phys. 2009, 11, 27672778.

[18] Chen, P.; Xu, W.; Zhou, X. C.; Panda, D.; Kalininskiy, A. Single-nanoparticle catalysis at single-turnover resolution. Chem. Phys. Lett. 2009, 470, 151-157.

[19] Edman, L.; Földes-Papp, Z.; Wennmalm, S.; Rigler, R. The fluctuating enzyme: A single molecule approach. Chem. Phys. 1999, 247, 11-22.

[20] Velonia, K.; Flomenbom, O.; Loos, D.; Masuo, S.; Cotlet, M.; Engelborghs, Y.; Hofkens, J.; Rowan, A. E.; Klafter, J.; Nolte, R. J. M.; de Schryver, F. C. Single-enzyme kinetics of CALB-catalyzed hydrolysis. Angew. Chem. Int. Ed. 2005, 44, 560-564.

[21] English, B. P.; Min, W.; van Oijen, A. M.; Lee, K. T.; Luo, G. B.; Sun, H. Y.; Cherayil, B. J.; Kou, S. C.; Xie, X. S. Everfluctuating single enzyme molecule: Michaelis-Menten equation revisited. Nat. Chem. Biol. 2006, 2, 87-94.

[22] Smiley, R. D.; Hammes, G. G. Single molecule studies of enzyme mechanisms. Chem. Rev. 2006, 106, 30803094.

[23] Roeffaers, M. B. J.; Sels, B. F.; Uji-i, H.; De Schryver, F. C.; Jacobs, P. A.; De Vos, D. E.; Hofkens, J. Spatially resolved observation of crystal-face-dependent catalysis by single turnover counting. Nature 2006, 439, 572-575.

[24] Sakamoto, M.; Tachikawa, T.; Fujitsuka, M.; Majima, T. Photoreactivity of as-fabricated Au clusters at the singlecluster level. J. Am. Chem. Soc. 2009, 131, 6-7.

[25] Tachikawa, T.; Majima, T. Exploring the spatial distribution and transport behavior of charge carriers in a single titania nanowire. J. Am. Chem. Soc. 2009, 131, 8485-8495.
[26] Satterfield, C. N. Heterogeneous catalysis in practice; McGraw-Hill Book Company: New York, 1980.

[27] Xie, X. S. Single-molecule approach to dispersed kinetics and dynamic disorder: Probing conformational fluctuation and enzymatic dynamics. J. Chem. Phys. 2002, 117, 11024-11032.

[28] Lu, H. P.; Xun, L. Y.; Xie, X. S. Single-molecule enzymatic dynamics. Science 1998, 282, 1877-1882.

[29] Kou, S. C.; Cherayil, B. J.; Min, W.; English, B. P.; Xie, X. S. Single-molecule Michaelis-Menten equations. J. Phys. Chem. B 2005, 109, 19068-19081.

[30] Min, W.; English, B. P.; Luo, G. B.; Cherayil, B. J.; Kou, S. C.; Xie, X. S. Fluctuating enzymes: Lessons from singlemolecule studies. Acc. Chem. Res. 2005, 38, 923-931.

[31] Min, W.; Gopich, I. V.; English, B. P.; Kou, S. C.; Xie, X. S.; Szabo, A. When does the Michaelis-Menten equation hold for fluctuating enzymes? J. Phys. Chem. B 2006, 110, 20093-20097.

[32] Xie, S. N. Single-molecule approach to enzymology. Single Mol. 2001, 2, 229-236.

[33] Cao, J. S. Event-averaged measurements of singlemolecule kinetics. Chem. Phys. Lett. 2000, 327, 38-44.

[34] Cao, J. S. Single molecule waiting time distribution functions in quantum processes. J. Chem. Phys. 2001, 114, 5137-5140.

[35] Witkoskie, J. B.; Cao, J. S. Single molecule kinetics. I. Theoretical analysis of indicators. J. Chem. Phys. 2004, 121, 6361-6372.

[36] Qian, H.; Elson, E. L. Single-molecule enzymology: Stochastic Michaelis-Menten kinetics. Biophys. Chem. 2002, 101-102, 565-576.

[37] Gopich, I. V.; Szabo, A. Theory of the statistics of kinetic transitions with application to single-molecule enzyme catalysis. J. Chem. Phys. 2006, 124, 154712.

[38] Xue, X.; Liu, F.; Ou-Yang, Z. -C. Single molecule Michaelis-Menten equation beyond quasistatic disorder. Phys. Rev. E 2006, 74, 030902.

[39] Chaudhury, S.; Cherayil, B. J. Dynamic disorder in single-molecule Michaelis-Menten kinetics: The reaction-diffusion formalism in the Wilemski-Fixman approximation. J. Chem. Phys. 2007, 127, 105103.

[40] Zhou, Y. J.; Zhuang, X. W. Kinetic analysis of sequential multistep reactions. J. Phys. Chem. B 2007, 111, 1360013610.

[41] Edman, L.; Rigler, R. Memory landscapes of singleenzyme molecules. Proc. Natl. Acad. Sci. USA 2000, 97, 
8266-8271.

[42] Flomenbom, O.; Velonia, K.; Masuo, S.; Loos, D.; Cotlet, M.; Engelborghs, Y.; Hofkens, J.; Rowan, A. E.; Nolte, R. J. M.; van der Auweraer, M.; de Schryver, F. C.; Klafter, J. Stretched exponential decay and correlations in the catalytic activity of fluctuating single lipase molecules. Proc. Natl. Acad. Sci. USA 2005, 102, 2368-2372.

[43] Antikainen, N. M.; Smiley, R. D.; Benkovic, S. J.; Hammes, G. G. Conformation coupled enzyme catalysis: Single-molecule and transient kinetics investigation of dihydrofolate reductase. Biochemistry 2005, 44, 1683516843.

[44] Gorris, H. H.; Rissin, D. M.; Walt, D. R. Stochastic inhibitor release and binding from single-enzyme molecules. Proc. Natl. Acad. Sci. USA 2007, 104, 1768017685.

[45] Shi, J.; Dertouzos, J.; Gafni, A.; Steel, D.; Palfey, B. A. Single-molecule kinetics reveals signatures of half-sites reactivity in dihydroorotate dehydrogenase $A$ catalysis. Proc. Natl. Acad. Sci. USA 2006, 103, 5775-5780.

[46] Zhang, Z. Q.; Rajagopalan, P. T. R.; Selzer, T.; Benkovic, S. J.; Hammes, G. G. Single-molecule and transient kinetics investigation of the interaction of dihydrofolate reductase with NADPH and dihydrofolate. Proc. Natl. Acad. Sci. USA 2004, 101, 2764-2769.

[47] Bagshaw, C. R.; Conibear, P. B. Single molecule enzyme kinetics: Application to myosin atpases. Biochem. Soc. Trans. 1999, 27, 33-37.

[48] Paige, M.; Fromm, D. P.; Moerner, W. E. Biomolecular applications of single-molecule measurements: Kinetics and dynamics of a single-enzyme reaction. Proc. Soc. Photo-Opt. Instrum. Eng. 2002, 4634, 92-103.

[49] Fersht, A. Structure and mechanism in protein science: A guide to enzyme catalysis and protein folding; W. $\mathrm{H}$. Freeman and Company: New York, 1998.

[50] van Oijen, A. M.; Blainey, P. C.; Crampton, D. J.; Richardson, C. C.; Ellenberger, T.; Xie, X. S. Singlemolecule kinetics of $\lambda$ exonuclease reveal base dependence and dynamic disorder. Science 2003, 301, 1235-1238.

[51] de Cremer, G.; Roeffaers, M. B. J.; Baruah, M.; Sliwa, M.; Sels, B. F.; Hofkens, J.; De Vos, D. E. Dynamic disorder and stepwise deactivation in a chymotrypsin catalyzed hydrolysis reaction. J. Am. Chem. Soc. 2007, 129, 1545815459 . 